\title{
Mujeres, acción y diferencia. Esbozos para un feminismo crítico
}

\author{
Women, action and difference. \\ Outlines for a critical feminism
}

\section{Marina Gorali ${ }^{1}$ \\ Universidad de Buenos Aires - Argentina}

Revista Derechos en Acción ISSN 2525-1678/ e-ISSN 2525-1686

Año 5/N 15, 0toño 2020 (21 marzo a 21 junio), 542-553

DOl: https://doi.org/10.24215/25251678e412

"Nunca se convierte ya en hoy mismo"

W. Benjamin

\section{Introducción}

¿Cómo pensar lo femenino? ¿Es posible ubicar allí algo del orden de lo identificable que permita inscribirnos como parte de un conjunto? ¿Cómo tramitar las diferencias? ¿Cómo articular una propuesta de acción que soporte lo inasible de la subjetividad misma? ¿Cómo impulsar las condiciones jurídicas que atribuyan

\footnotetext{
1 Doctora en Derecho por la Universidad de Buenos Aires. Abogada - Facultad de Derecho UBA. Licenciada en Ciencia Política - Facultad de Ciencias Sociales UBA. Investigadora del proyecto Ubacyt Modalidad I "Leer el derecho" dirigido por el Dr. Cárcova. Profesora Adjunta de Filosofía del Derecho UBA a cargo de los cursos del CPO "Lecturas contemporáneas acerca del derecho" y "El conocimiento del derecho", Cátedra Dra. Ruiz, Facultad de Derecho, UBA. Profesora de Sociología jurídica de la Universidad Nacional de Avellaneda (UNDAV). El abordaje de las asignaturas tiene por objeto efectuar aportes formativos críticos con apoyo en la semiótica jurídica que sirvan de base para la comprensión y articulación de acciones frente a problemas jurídicos contemporáneos (acceso a la justicia, violencias, género, justicia y construcción social de la memoria, opacidad del derecho, migraciones, interpretación judicial, pluralismos jurídicos, diferencia e interculturalidad). (ORCID: 0000-0002-720-5699). Contacto: marinagorali@derecho.uba.ar//mgorali@undav.edu.ar.
} 
un rol activo al Estado en la protección de los derechos de las mujeres frente a la violencia estructural? ¿Cómo desplazar esta esfera de inscripción moderna de la violencia sacrificial que produce día a día cada vez más cuerpos desechables? El presente trabajo pretende abordar estos interrogantes dando cuenta de la necesidad de forjar un feminismo crítico que sitúe los discursos de género lejos de toda lectura fácil y simplista. Este desafío supone consolidar un movimiento feminista que problematice $\mathrm{y}$ desarticule ciertas categorías medulares de la teoría jurídica de nuestra modernidad occidental, categorías que:

a) han imposibilitado comprender la complejidad y desplazamientos de los procesos de constitución subjetiva,

b) han ignorado las micropolíticas de la diferencia y las disputas/ renegociaciones que éstas implican,

c) han presupuesto una temporalidad teleológica como marco de acción,

d) no han dado cuenta cómo los paradigmas de excepción se han convertido en formas paradigmáticas de gobierno, constituyéndose en una de las esferas de inscripción moderna de la violencia sacrificial.

Se trata, en definitiva, de habilitar un modo de pensar los discursos de género que permita reinscribir la crítica no como develamiento de una supuesta "verdad esencial" oculta sino como una praxis que transforme a partir de los propios límites que el orden simbólico instituye. Esto es, entender que toda conciencia social se constituye como (y en) una cadena semiótica. Circunstancia que exige interrogar la pretensión de hallar una conciencia (en este caso de la mujer) en estado positivo y puro. En todo caso, la posibilidad de la acción radica precisamente en sustraerse a esa pretensión de corte positivista desorganizando ese objeto y re-eslabonando la cadena sígnica. Esto supone asimismo desplazar la noción de sujeto unificado/ identidad original. El sujeto es, ante todo, el sujeto de una carencia, de una falta. Como resultado, cualquiera sea la identidad que tenga, se constituye mediante actos de identificación y lenguaje. 
En este sentido, Chantal Mouffe advierte sobre la necesidad de desplazar las identidades sustancializadas como una condición fundamental para la formulación de una política feminista:

Para las feministas comprometidas (...).sólo cuando descartemos la visión del sujeto como un agente al mismo tiempo racional y transparente para sí mismo, y descartemos también la supuesta unidad y homogeneidad del conjunto de sus posiciones, tendremos posibilidades de teorizar la multiplicidad de las relaciones de subordinación. (Mouffe, 1999, p. 5)

\section{Identidad narrativa y diferencia}

En La Condición humana Arendt (1958) escribe "La cualidad específica de revelación de la acción y la palabra, la manifestación implícita del sujeto que actúa y habla, está tan indisolublemente ligada al flujo vivo del actuar y el hablar que sólo puede ser representada (...) por medio de la mimesis" (p.210). La revelación del "quien" supone así una política de la narración. Para Arendt, el relato, en tanto expresión de la acción narrada, se constituye como el lugar de realización del pensamiento esencialmente político. En este sentido, la acción prevalece bajo la condición de su narratividad, de ser una acción narrada. La acción individual no constituye pues la acción maravillosa, sólo se vuelve tal en la medida en que sea memorable. Son las y los espectadores los que hacen de la polis una organización creadora de memoria o de historia. Para que una historia se convierta en una historia contada debe existir, en primer lugar, un inter-est (entre-dos) por el cual adquieran forma la memoria y el testimonio. Es entonces por el relato como se realiza el pensamiento esencialmente político. El relato es la dimensión inicial en la cual viven los hombres y las mujeres, la dimensión de un bios y no de una zoé. El relato es la acción más inmediatamente compartida y en tal sentido la más inicialmente política. Este aspecto será retomado también por P. Ricoeur. Renunciando a toda pretensión del sujeto por 
fundarse y conocerse a sí por "sí mismo", Ricoeur entiende que toda comprensión de sí debe ir mediatizada por el análisis de los signos, símbolos y textos. Este criterio para deducir la identidad de yo como identidad simbolizada es ampliado en "Tiempo y Narración" en el sentido de una identidad narrada. Allí nos invita a pensar en la narración como la clave de la identificación subjetiva. La historia narrada dice el quién de la acción. La identidad del quién no es, pues, ella misma más que una identidad narrativa. Hablar de identidad narrativa es reconocer la incapacidad del sujeto de captarse instantáneamente. Ricoeur supera así una visión sustancialista del sujeto: sin narración no hay identificación posible ni del individuo ni de las comunidades. Identificación que se producirá en el ámbito práctico del relato de sus actos.

¿Cómo pensar entonces lo femenino en el marco de estas reflexiones? En primer lugar, advertir la importancia de interrogar los discursos que presuponen un núcleo homogéneo de una verdad transparente del "ser mujer". Estos discursos suelen sostenerse sobre la base de presupuestos sustancialistas o bien del naturalismo positivista. Lejos de ello, las construcciones subjetivas que convergen en el significante "mujer" se constituyen mediante complejos actos de identificación y lenguaje. Identificaciones que desplazan y reinscriben posiciones en campos de pertenencia cuyas fronteras son siempre móviles y sujetas a tensiones y disputas. La distinción entre exterior e interior no es necesariamente delimitable ya que éstas implican fórmulas de diferenciación que tramitan esos límites. Esto supone comprender también que los propios grupos "identitarios" constituyen mecanismos por los cuales establecen formas de exclusión e inclusión en diversos planos de diferencia. Los rituales, en este sentido, constituyen un momento privilegiado donde es posible advertir el despliegue y renegociación de estas tensiones.

Las estadísticas sobre femicidios constituyen un espacio que da cuenta de ello. Contar es, ante todo, un acto político. Resulta, en este sentido, imprescindible sostener políticas de censo y registro que amplíen la propia categoría de "femicidio". 
Circunstancia que supone no sólo contabilizar, sino interrogar la propia categoría de mujer. Esto implica asimismo la obligación del Estado de proteger los derechos de todas las mujeres frente a la violencia estructural. Responsabilidad amparada por el propio bloque constitucional, ${ }^{2}$ que demanda del Estado ya no un mero rol neutral o pasivo sino, por el contrario, exige un papel activo en la prevención y protección de grupos sometidos a procesos estructurales de discriminación y violencia.

En un nivel más complejo, es preciso comprender también que la diferencia sexual puede ser pensada (se juega) en el plano de lo que el estructuralismo denomina "la posición". Como bien explica Deleuze (1982), no se trata de un sitio en una extensión real, ni de lugares en extensiones imaginarias, sino de sitios y lugares en un espacio propiamente estructural, es decir topológico. La estructura es en sí misma un sistema de elementos y de relaciones diferenciales. Ella es diferencial en sí misma, y diferenciadora en su efecto. Mientras que lo imaginario tiende a reflejar y a reagrupar sobre cada término el efecto total de un mecanismo de conjunto, la estructura simbólica asegura la diferenciación de los términos y la diferenciación de los efectos.

Ahora bien, ¿cómo se organizan estas posiciones? ¿Qué las distribuye? Contra el carácter dual de la imaginación -explica Deleuze- el Tercero interviene en el sistema simbólico, distribuyendo las series, desplazándolas relativamente, haciéndolas comunicar y sobre todo, impidiendo a la una abatirse imaginariamente sobre la otra. "Deuda, carta, pañuelo o corona", la naturaleza de este objeto es siempre desplazado en relación consigo mismo.

Tiene como propiedad no estar donde se lo busca, pero en revancha también la de ser encontrado donde no está". Se dirá que "falta en su lugar" (...)También, que falta en su propia semblanza (...) que falta a su propia

\footnotetext{
2 Cfr. Abramovich, V. "Responsabilidad estatal por violencia de género: comentarios sobre el caso "Campo Algodonero" en la Corte Interamericana de Derechos Humanos", Anuario de Derechos Humanos, 2010. pp. 167-182.
} 
identidad. Es en este sentido que el desplazamiento, y más generalmente todas las formas de cambio, no forma un carácter añadido desde afuera, sino la propiedad fundamental que permite definir la estructura como orden de los lugares bajo la variación de las relaciones. (Deleuze, 1982, p.9)

Toda la estructura es movida por aquel objeto que es, ante todo, una casilla vacía, que falta en su propio origen. Distribuyendo las diferencias en toda la estructura, haciendo variar las relaciones diferenciales con sus desplazamientos, el objeto $=x$ constituye el diferenciante de la diferencia misma.

¿Qué implicancias tiene ello a nivel de los discursos de género? Fundamentalmente pone en evidencia la imperiosa necesidad de admitir el carácter incompleto de la subjetividad misma. Esta incompletitud constitutiva muestra cómo la diferencia sexual lejos de ser un conjunto firme de oposiciones simbólicas estáticas, es el nombre de "una incógnita" que resiste cualquier simbolización. Pero es precisamente esta imposibilidad la que (paradójicamente) da lugar a la posibilidad misma de la lucha por el significado de la "diferencia sexual". ${ }^{3}$ Circunstancia que exige repensar no sólo las políticas y estrategias de acción sino, ante todo, la idea misma de acción (y la temporalidad que ella trae aparejada).

\section{Género, tiempo y acción}

En "El tiempo de las mujeres" Kristeva (1979) sitúa la problemática de las mujeres en una interrogación sobre el tiempo: aquel que el movimiento feminista hereda, aquel que su aparición modifica. En este sentido ubica dos momentos del movimiento feminista: el primero, universalista en su trayectoria, globaliza los problemas de las mujeres de diferentes edades, culturas, situación socio-económica bajo la etiqueta de la Mujer

3 Ver el debate entre Judith Butler, Ernesto Laclau y Slavoj Zizek en Contingencia, hegemonía, universalidad. Diálogos contemporáneos en la izquierda. Buenos Aires, FCE, 2000. 
Universal. Frente a esta primera corriente, que se reconocería en una concepción del tiempo lineal y teleológico -el tiempo del proyecto-; es posible ubicar una nueva generación de mujeres que llegaron al feminismo tratando de dar lenguaje a las especificidades y experiencias culturales y corporales que la generación anterior dejó mudas. Al exigir el reconocimiento de una singularidad irreductible y resplandeciente en sí misma, plural, fluida, no idéntica, este feminismo se situaría -a criterio de la autora- fuera del tiempo lineal de las identidades.

Creo que es precisamente en este pasaje, en esta torsión, donde es necesario inscribir la posibilidad misma de la acción: en un cuestionamiento profundo de la instrumentalidad y la linealidad temporal como modo de pensar la transformación. Vuelvo a Arendt (1957): "La historia real en que estamos comprometidos mientras vivimos no tiene ningún autor visible o invisible, porque no está fabricada". (p.105) Al modo de la Jetztzeit ${ }^{4}$ benjaminiana o del teatro de Brecht, el actuar interviene ya no bajo el modo del cálculo sino de la interrupción ${ }^{5}$. H. Bhabha explica que en Brecht, la interrupción es el método dramático que posibilita precisamente la ruptura del encantamiento al que el naturalismo teatral y la normalización someten al público (Bhabha, 2013, 38). Es en esta doble función de apertura e interrupción que, entiendo, la acción exhibe su capacidad propiamente transformadora. Como bien dice Benjamin apropósito del estreno de la obra "La madre" de Brecht "La dialéctica no necesita de las nebulosas lejanías: su casa son las cuatro paredes de la praxis, y ahí, puesta en pie en el umbral del instante, dice las palabras con las que acaba "La madre": "Nunca se convierte ya en hoy mismo". (Benjamin, 1932, 118) Es en definitiva Pelagia Vlásova, doblemente explotada en tanto trabajadora pero también en tanto mujer y madre, quien porta

4 El término "Jetztzeit" se lo suele traducir como "tiempo-ahora".

5 La imponente Movilización por el derecho al aborto legal seguro y gratuito durante el tratamiento del proyecto en la Cámara de Diputados de la Nación, muestra la capacidad propiamente transformadora de la acción. Lo que se ha dado en llamar la "Revolución de la hijas". 
la posibilidad misma de la revolución. La función materna lejos ya de ser denunciada como cómplice es, al decir de Benjamin, "la praxis hecha carne". O dicho en otras palabras: el agente de la transformación.

\section{Simbolismo y redistribución: un falso dilema}

Suele leerse a menudo una cierta preocupación por parte de algunas/os autores ${ }^{6}$ por las consecuencias que los discursos de la diferencia vendrían a provocar en relación a una supuesta "suspensión silenciosa de los análisis de clase". Suspensión que reduciría la economía a un sitio discursivo, renaturalizando al capitalismo como una consecuencia inadvertida.

En esta misma línea, Nancy Fraser acusa a cierto feminismo -formado a la luz del giro lingüístico- de haber atenuado toda pretensión de transformación social dirigiendo sus energías más en valorizar la diferencia que en promover la igualdad económica. (Fraser, 2015, p.17)

Estas afirmaciones requieren una serie de consideraciones:

En primer lugar (yendo a los dichos de Fraser) cabe interrogar si es posible disociar epistemológicamente "producción" de "cultura". Distinción que sólo una lectura esquemática de Marx podría reivindicar. Como bien señala Foucault (1976) "Marx es demasiado astuto como para admitir esto". (p.30) Un lúcido marxista como E. Gruner (2006) explica que la crítica supone necesariamente una cierta teoría de lo simbólico. El ser humano se relaciona con (y organiza) su realidad a través de la mediación simbólica (empezando por el propio lenguaje). La "interpretación" (crítica) consistiría así -para el autor- en interrogar críticamente esas construcciones simbólicas para mostrar -incluso para producir- sus vacíos, sus "agujeros de sentido" (puesto que no son verdades eternas, nunca están plenamente completas, no pueden explicarlo todo), y entonces construir,

6 Wendy Brown, Salvoj Zizek, entre otros. 
producir, un sentido nuevo sobre esos "blancos" o ausencias. Por ello el autor insiste en que Marx no interpreta a la "sociedad burguesa": lo que Marx interpreta es la interpretación "burguesa" de la sociedad, es decir, la construcción simbólica que la "burguesía" ha producido sobre su propia praxis. (Gruner, 2006, p.124)

Por otro lado, esta pretendida dicotomía entre "redistribución" y "políticas de la diferencia" (una -la segunda- implicaría un supuesto abandono de la otra -la primera.) olvida que los propios conceptos de clase, producción, economía, ponen en juego significantes que, en modo alguno, se encuentran fuera de disputa. Por el contrario, expresan -inscriben- el espacio mismo de la lucha. Como explica Voloshinov, todo producto ideológico aparece como signo que se constituye como "la arena de la lucha social". (Voloshinov, p.47) En este sentido, para el autor, un signo sustraído de la tensa lucha social se convierte en algo muerto, sin vida.

\section{Hacia un Feminismo crítico}

Reinscribiendo lo señalado, necesitamos forjar perspectivas críticas que:

a) interroguen los discursos que presuponen un núcleo homogéneo de una verdad transparente del "ser mujer";

b) desplacen las identidades sustancializadas como una condición fundamental para la formulación de una política feminista que permita teorizar la multiplicidad de las relaciones de subordinación;

c) asuman que las construcciones subjetivas que convergen en el significante "mujer" se constituyen mediante complejos actos de identificación y lenguaje. Identificaciones que desplazan y reinscriben posiciones en campos de pertenencia cuyas fronteras son siempre móviles y sujetas a tensiones y disputas;

d) den voz a una nueva generación de mujeres que llegaron al feminismo tratando de dar lenguaje a las especificidades y 
experiencias corporales y culturales que la generación anterior dejó mudas;

e) sostengan políticas que interroguen la propia categoría de mujer;

f) admitan el carácter incompleto de la subjetividad misma. Incompletitud que muestra cómo la diferencia sexual lejos de ser un conjunto firme de oposiciones simbólicas estáticas, es el nombre de "una incógnita" que resiste cualquier simbolización;

g) comprendan que es precisamente esta imposibilidad la que (paradójicamente) da lugar a la posibilidad misma de la lucha por el significado de la "diferencia sexual;

h) den cuenta cómo los paradigmas de excepción se han convertido en la regla, constituyéndose como una de las esferas de inscripción moderna de la violencia sacrificial que produce día a día cada vez más cuerpos desechables;

i) demanden del Estado un rol activo en la protección de los derechos de las mujeres frente a la violencia estructural;

j) sitúen la acción en un cuestionamiento profundo de la instrumentalidad y la linealidad temporal como modo de pensar la transformación;

k) entiendan que la crítica supone necesariamente una cierta teoría de lo simbólico. Circunstancia que, en modo alguno, supone abandonar la lucha por la inclusión social y las igualdades económicas. Por el contrario, permite comprender que la relación con las posiciones de clase están siempre mediadas por el lenguaje. Lenguaje que se constituye no sólo como sistema de diferencias sino como la arena misma de la disputa social.

Es, en definitiva, asumiendo estas complejidades y paradojas que podrán aproximarse líneas de reflexión crítica que operen como marco y soporte de una posible transformación. Transformación que permita consolidar un feminismo crítico que porte la igualdad y la diferencia como voces de una misma articulación. 


\section{Referencias bibliográficas}

Arendt, H. (1957) Labor, trabajo, acción. Una conferencia. En De la bistoria a la acción, Buenos Aires: Paidos.

- (1958) La condición bumana, Buenos Aires: Paidós.

Babba, H. (2013) Reconocimiento, derechos y vecindad: hacia una ética de las comunidades paradójicas. En Nuevas minorías, nuevos derechos, Buenos Aires: Siglo XXI.

Benjamin, W. (1932) Un drama familiar en el teatro épico. Con ocasión del estreno de La madre de Brecht. En Walter Benjamin, Obras, Libro II, Madrid: Abada Editores.

Brown, W. (1995) States of injury. New Jersey: Princeton University Press.

Butler, J. Zizek, S. Laclau, E. (2000) Contingencia, hegemonía, universalidad. Diálogos contemporáneos en la izquierda. Madrid: FCE.

Cárcova, C. M. (2014) Racionalidad formal o racionalidad hermenéutica para el derecho en las sociedades complejas Revista Jurisprudencia Argentina, Número especial, Los derechos fundamentales en la Constitución: interpretación y lenguaje. Buenos Aires: Abeledo Perrot, pp. 13-26.

Deleuze, G. (1982) En qué se reconoce el estructuralismo. En " $L a$ isla desierta y otros textos", Valencia: Editorial Pre-textos.

Foucault, M. (2005) Redes de poder. En Ferrer, Christian El lenguaje libertario: antología del pensamiento anarquista contemporáneo, La Plata: Terramar.

Fraser, N. (2015) Fortunas del feminismo. Del capitalismo gestionado por el Estado a la crisis neoliberal. Madrid: Traficantes de sueños.

Gorali, M. (2014) Derecho, comunidad política e interpretación. En Cárcova, C. M. Los derechos fundamentales en la Constitución: interpretación y lenguaje. Revista Jurisprudencia Argentina, Número especial, Buenos Aires: Abeledo Perrot, pp. 33- 40.

- (2015) Derecho y Estructuralismo: algo de lo que es preciso hablar en voz alta. Revista digital Carrera Docente, Año IV, No 6, Facultad de Derecho, UBA, pp.31-42. 
Gruner, E. (2006) Lecturas culpables. Marx(ismos) y la praxis del conocimiento. En La teoría marxista hoy. Problemas y perspectivas, Buenos Aires: CLACSO.

Kristeva, J. (1979) El tiempo de las mujeres. Revista 34/44, Universidad Paris VII, No 5, pp 5-19.

- (1999) El genio femenino: Hannab Arendt. Buenos Aires: Paidos.

Lamas, M. (1994) Cuerpo: diferencia sexual y género. Debate Feminista, $\mathrm{N}^{\mathrm{o}} 10$, México.

Merry, S. E. (2001) Las mujeres, la violencia y el sistema de derechos humanos. En Margorie Agosin (ed.) Women, Gender, and Human Rights. A Global Perspective, Londres: Rutgers University Press.

Mouffe, Ch. (1999) Feminismo, ciudadanía y política democrática radical. En Lamas, M. (comp) Ciudadanía y feminismo, Mexico: Instituto Federal Electoral.

Ricoeur, P. (1985) Tiempo y Narración I. Configuración del tiempo en el relato histórico. Buenos Aires: Siglo XXI.

Ruiz, A. (2001) Idas y vueltas por una teoría crítica del derecho. Buenos Aires, Editores del Puerto Departamento de Publicaciones de la Facultad de Derecho de la UBA.

Sneh,P y Cosaka, J. (2000) Del lenguaje del exterminio al exterminio del discurso. La shoa en el siglo. Buenos Aires: Xavier Bóveda.

Spivak, G. (1997) Estudios de la subalternidad: deconstruyendo la historiografía. En Debates Post Coloniales, La Paz: Aruwiyiri historias.

Voloshinov, V. (1929) El marxismo y la filosofía del lenguaje. Buenos Aires: Ediciones Godot, 2009. 